\title{
Long-lived multilayer coatings for smart windows: integration of energy-saving, antifogging and self-healing functions
}

Lin $\mathrm{Yao}^{\mathrm{a}}$, Zhe Qu ${ }^{\mathrm{a}, \mathrm{b}}$, Rui Sun ${ }^{\mathrm{a}, \mathrm{c}}$, Zili Pang ${ }^{\mathrm{a}}$, Ying Wang,d, Binbin Jin ${ }^{\mathrm{a}, \mathrm{d}}$, Junhui Hea,

a Functional Nanomaterials Laboratory, Center for Micro/Nanomaterials and Technology, and Key Laboratory of Photochemical Conversion and Optoelectronic Materials, Technical Institute of Physics and Chemistry, Chinese Academy of Sciences, Zhongguancundonglu 29, Haidianqu, Beijing 100190, China.

$\mathrm{b}$ The Affiliation Key Laboratory of Coal Science and Technology of Shanxi Province and Ministry of Education, Taiyuan University of Technology, Taiyuan 030024, Shanxi, China.

${ }^{c}$ School of Chemical and Environmental Engineering China University of Mining and Technology (Beijing), Beijing, 100083, China.

d University of Chinese Academy of Sciences, Beijing 100049, China.

* Corresponding author. Fax: +86 10 82543535. E-mail address: jhhe@mail.ipc.ac.cn. 


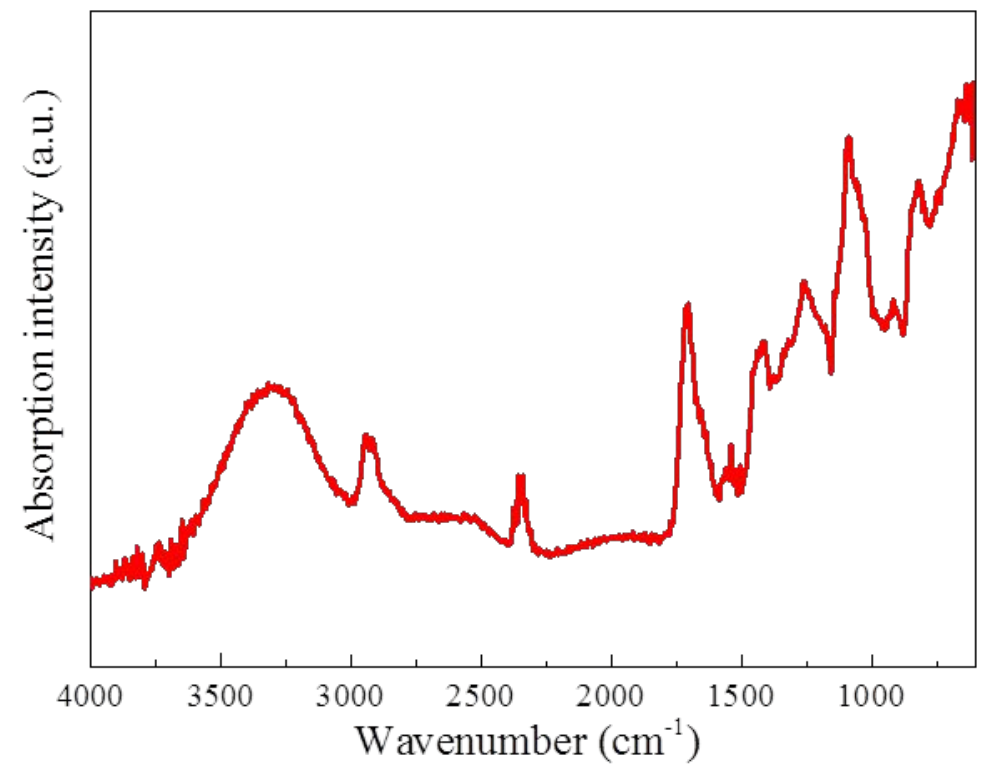

Figure S1. FTIR spectra of the polymer thin film.

\section{Friction force between coated substrate and sandpaper}

As shown in Figure S1, an electric device pulls a combination of the coated substrate (5 g) and a weight $(100 \mathrm{~g})$, which are put on a piece of sandpaper, in a uniform motion. The $\mathrm{f}_{\text {traction }}(0.2 \mathrm{~N})$ could be read out by the spring-loaded thrust meter, and according to force equilibrium (Figure $\mathrm{S} 1), \mathrm{f}_{\text {friction }}$ was also $0.2 \mathrm{~N}$.

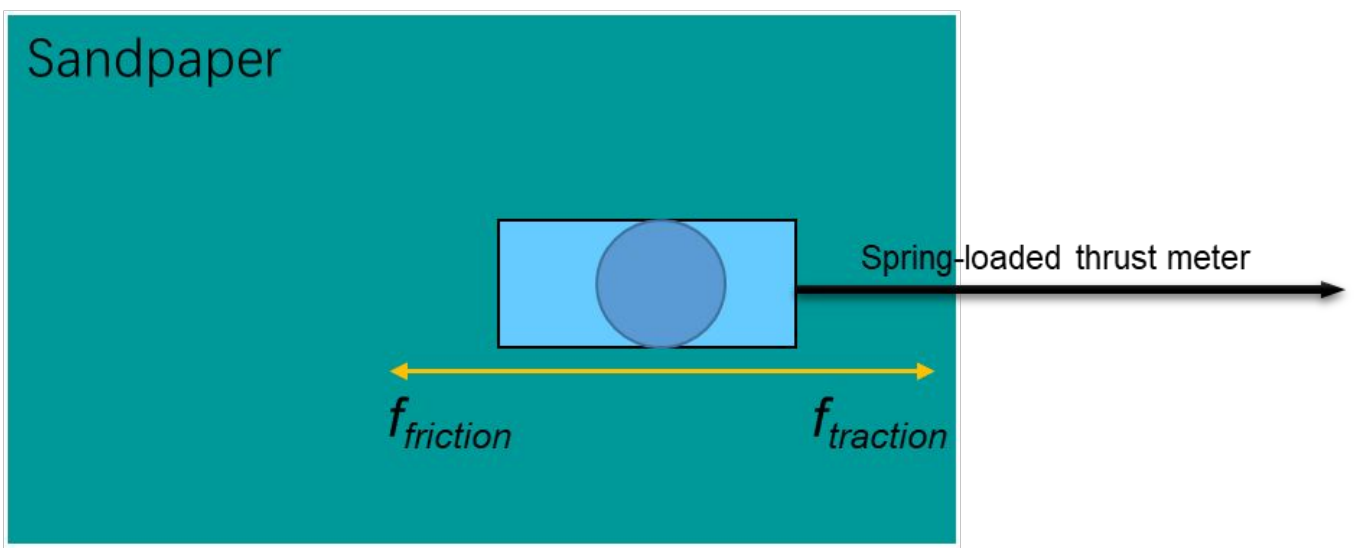

Figure S2 Top-view of the experimental setup and forces analysis of the coated substrate and weight. 

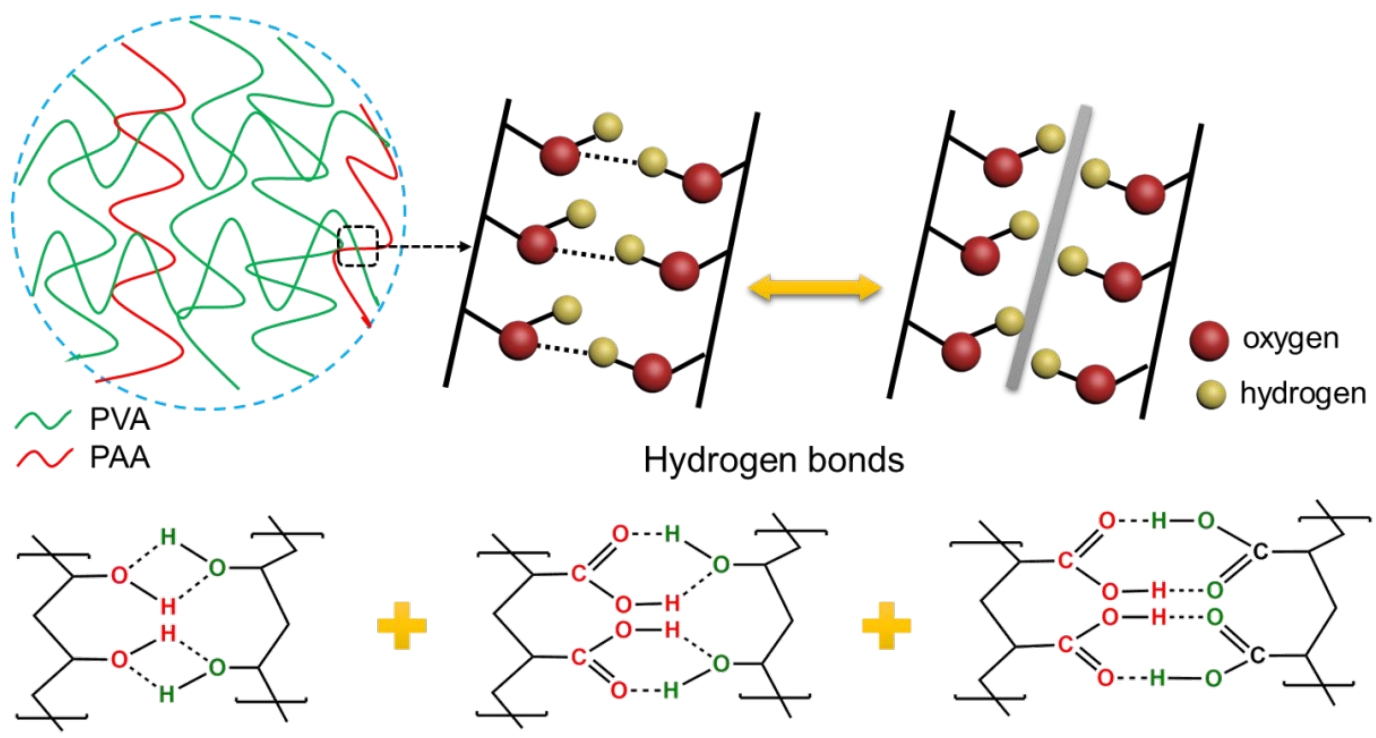

Figure S3. Schematic illustration of self-healing mechanisms of the HSi/V/FSi/P coating. 


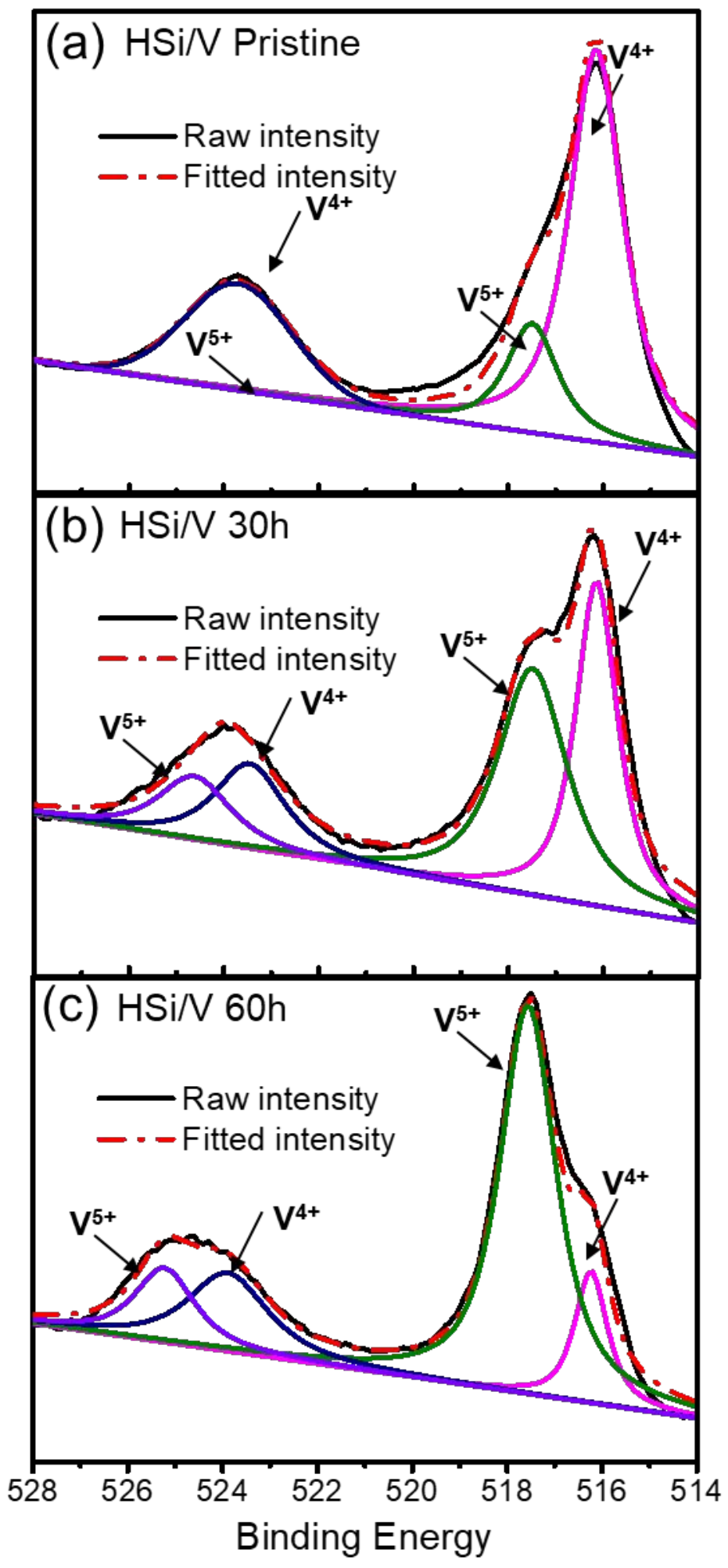

Figure S4. XPS measurements. V 2p core-level photoelectron spectra for HSi/V after different durations. 

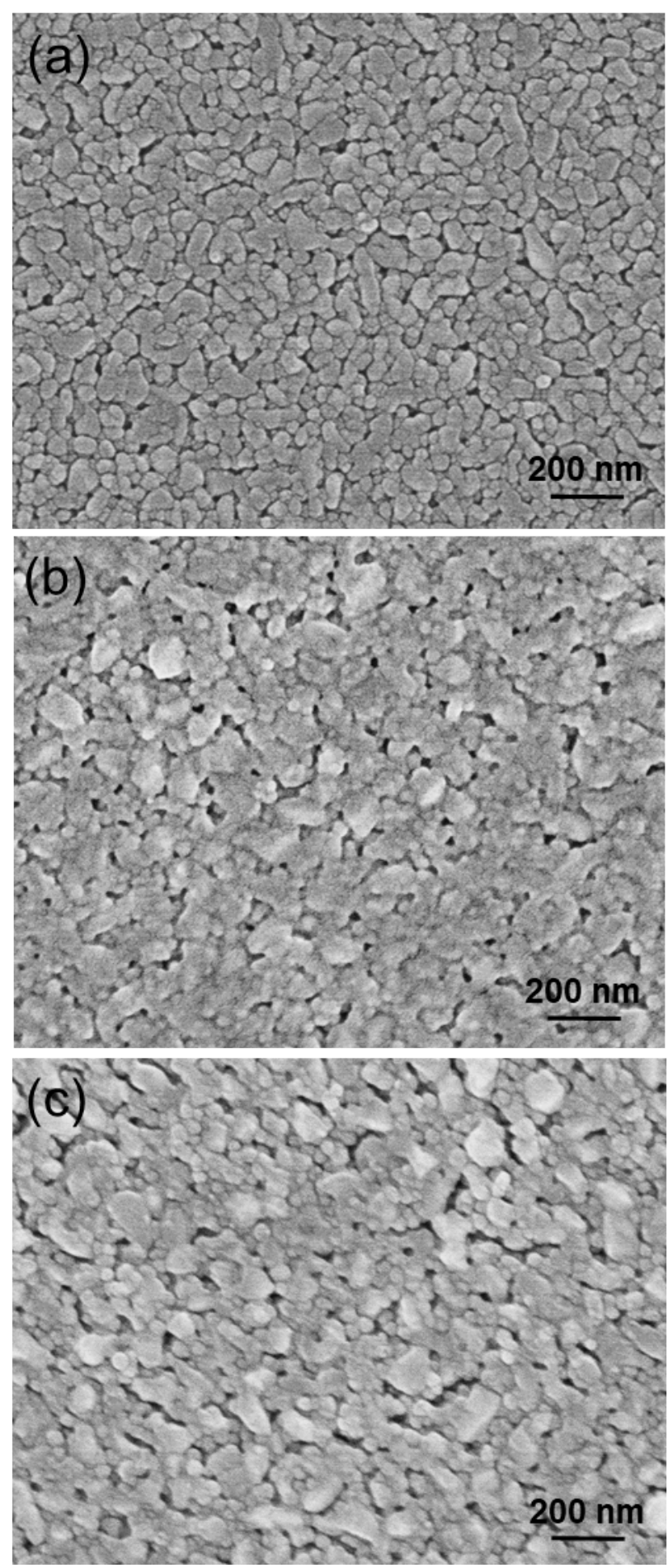

Figure S5. Top-view SEM images of HSi/V coating after different durations, (a) pristine, (b) $30 \mathrm{~h}$ and (c) $60 \mathrm{~h}$. 


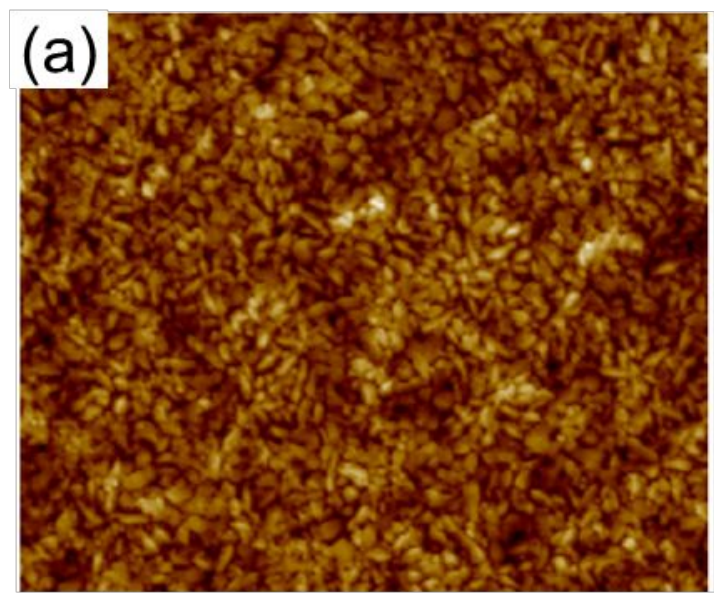

\section{$40 \mathrm{~nm}$}
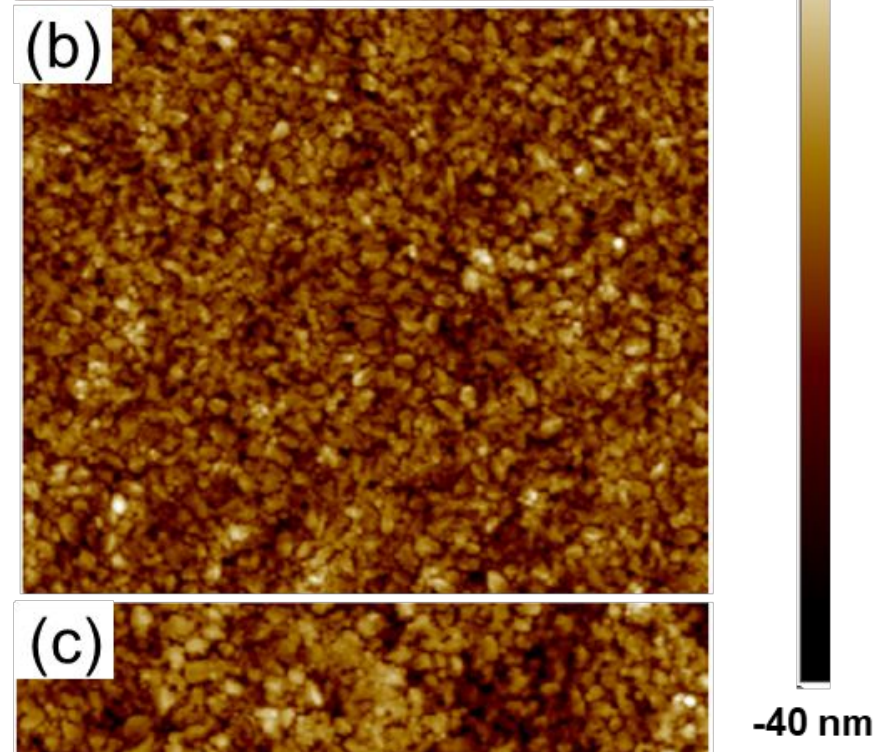

Figure S6. AFM images of HSi/V coating after different durations, (a) pristine, (b) $30 \mathrm{~h}$ and (c) 60 h. 

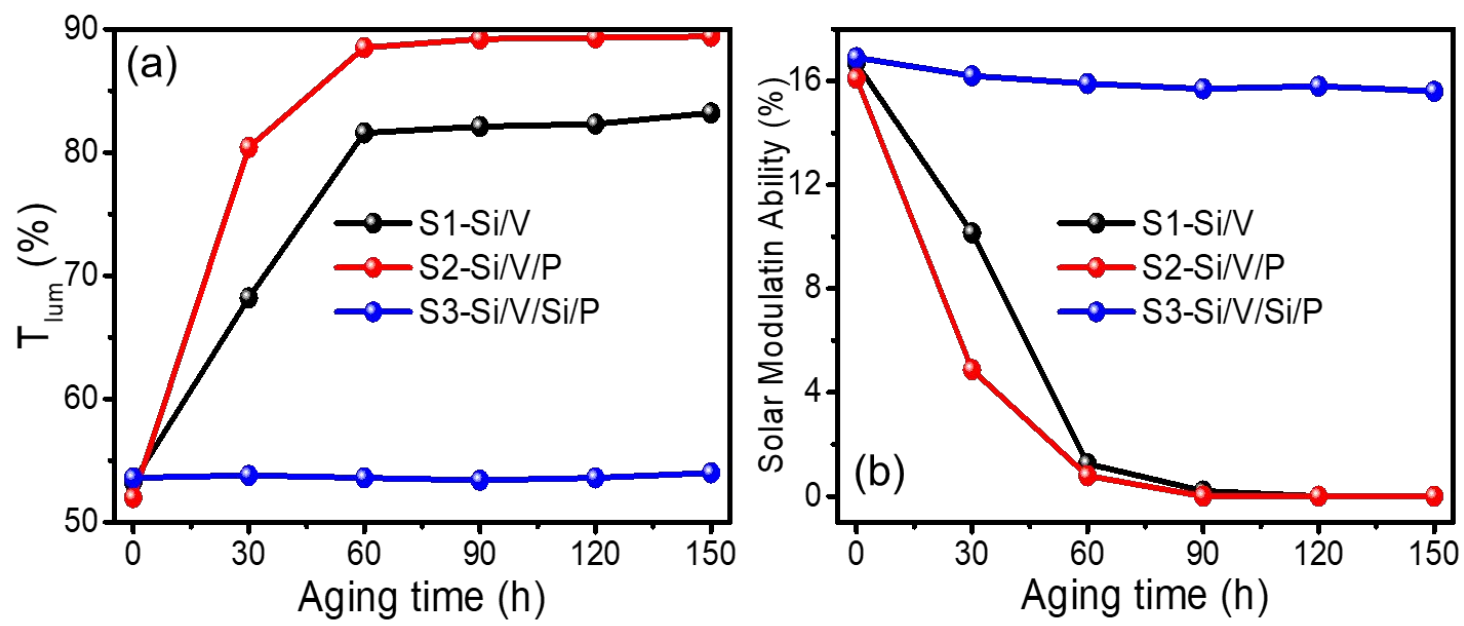

Figure $\mathrm{S} 7$. (a) $\mathrm{T}_{\text {lum }}$ and (b) $\Delta \mathrm{T}_{\text {sol }}$ of $\mathrm{S} 1, \mathrm{~S} 2$ and $\mathrm{S} 3$ coatings as a function of ageing duration.

Table S1. Optical properties of samples with $\mathrm{V}, \mathrm{V} / \mathrm{FSi}, \mathrm{V} / \mathrm{FSi} / \mathrm{P}$ and $\mathrm{HSi} / \mathrm{V} / \mathrm{FSi} / \mathrm{P}$ coatings, respectively.

\begin{tabular}{cccc}
\hline Sample & $\mathrm{T}_{\text {lum }}$ & $\Delta T_{\text {sol }}$ & $\Delta T_{I R}$ \\
\hline $\mathrm{V}$ & 34.7 & 10.5 & 25.1 \\
$\mathrm{~V} / \mathrm{FSi}$ & 33.6 & 9.6 & 19.8 \\
$\mathrm{~V} / \mathrm{FSi} / \mathrm{P}$ & 36.5 & 8.5 & 17.5 \\
$\mathrm{HSi} / \mathrm{V} / \mathrm{FSi} / \mathrm{P}$ & 54.0 & 16.4 & 31.1 \\
\hline
\end{tabular}

